\title{
Evidence for a low-penetrant extended phenotype of RTPS1 from a kindred with gain of SMARCB1 exon 6
}

\author{
Uwe Kordes $^{1}$, Victor Mautner ${ }^{2}$, Florian Oyen ${ }^{3}$, Christian Hagel ${ }^{1}$, Christian Hartmann ${ }^{4}$, \\ Michael Heuser ${ }^{5}$, Michael Frühwald ${ }^{6}$, Martin Hasselblatt ${ }^{7}$, Kathrin Oehl-Huber ${ }^{8}$, Reiner \\ Siebert $^{8}$, Reinhard Schneppenheim ${ }^{3}$, and Ulrich Schüller ${ }^{9}$ \\ ${ }^{1}$ University Medical Center Hamburg Eppendorf \\ ${ }^{2}$ Universitätsklinikum Hamburg-Eppendorf \\ ${ }^{3}$ University Hospital Medical Center \\ ${ }^{4}$ Institute of Pathology, Section of Neuropathology \\ ${ }^{5}$ Hannover Medical School \\ ${ }^{6}$ University Hospital Augsburg \\ ${ }^{7}$ University Hospital Münster \\ ${ }^{8}$ Ulm University and Ulm University Medical Center \\ ${ }^{9}$ Universitatsklinikum Hamburg Eppendorf Institut fur Neuropathologie
}

February 15, 2021

\begin{abstract}
We report on a long-term survivor of an atypical teratoid/rhabdoid tumor (ATRT-TYR) as an index patient, who carries a SMARCB1 exon 6 gain inherited from his father. The father was diagnosed with an unusual sequence of a myxopapillary INI1negative ependymoma and a relapsing BRAF V600 wild type hairy-cell leukemia. He has two yet healthy sisters aged 33 and 38 years carrying the same variant, from which one had lost an infant to a malignant brain tumor. This family highlights the existence of RTPS1-associated SMARCB1 germline alterations with reduced penetrance and extends the spectrum of involved diseases
\end{abstract}

\section{Introduction}

Pertubations of the SWI/SNF complex are common oncogenic events. The acquired loss of its core subunit member SMARCB1 drives an expanding spectrum of intra- and extracranial tumors, some characterized by rhabdoid features including atypical teratoid/rhabdoid tumor (ATRT), and others without such features, e. g. cribriform neuroepithelial tumor (CRINET), poorly differentiated chordoma, desmoplastic myxoid tumor, sinunasal undifferentiated carcinoma, epithelioid sarcoma, and renal medullary carcinoma. Distinct heterozygous SMARCB1 germline mutations cause Rhabdoid Tumor Predisposition Syndrome Type 1 (RTPS1), schwannomatosis, or Coffin-Siris syndrome. Although overlap has been reported, a genotype phenotype correlation exists with certain proximal and intronic mutations linked to schwannomatosis, distal missense mutation to Coffin-Siris, and truncating mutations to RTPS1 ${ }^{1}$.

\section{Methods and results}

The index patient III.1 (Figure 1A) was diagnosed with a non-metastatic posterior fossa brain tumor at the age of 6 years. Histology was consistent with an ATRT. After gross total tumor resection and treatment with radio-chemotherapy according to the EURHAB recommendations, the patient has remained in complete remission for 15 years after diagnosis. His father II.2 was diagnosed with a BRAF V600 wild-type hairy cell 
leukemia variant (HCL-v, with co-expression of CD19, CD103, CD11c, CD25) at the age of 47 years. He achieved a complete remission after 5 cycles of cladribin, but suffered from a relapse 9 years later, with a very good partial response to additional cycles of cladribin. With 54 years he underwent surgery for an L2-3 spinal ependymoma. At age 57 a hemi-colectomy for steroid-resistant ulcerative colitis was performed, apparently unrelated to his other malignoma.

Sanger sequencing of SMARCB1 did not detect any mutations in peripheral blood lymphocytes (PBL) of the index patient III.1(Figure 1B). However, a pattern suggesting gain of one copy of exon 6 of SMARCB1 was found by multiplex ligation-dependent probe amplification (MLPA). MLPA detected an apparently identicalSMARCB1 exon 6 gain in PBL of his father II.2 and two of his sisters III.2, III.4. Targeted next generation sequencing (NGS) confirmed the absence of a single nucleotide variant or small indel but could not identify the genomic integration site of the gained exon 6 sequences. SMARCB1 mutations were absent in PBL of the index patient's grandmother I.1, mother II.1, and of another sister III.3 . No material was available from a cousin IV.1, who had died from surgical complications of a malignant brain tumor at the age of 8 months. The index patient's grandfather I.2 had died from non-malignant disease and could not be tested. In the ten children of the grandfather I.2, a diagnosis of a malignancy was only known in II.2 . No detected carrier had any overt intellectual impairment, except for a learning disability in III.1 attributed to leukencephalopathy after intensive multi-modal treatment at young age. High resolution spinal and lower extremity MRI in III.1 and III.2 did not detect dorsal nerve root or peripheral nerve schwannoma.

BAF47 staining was negative in the ATRT of III.1 and in the myxopapillary ependymoma of II.1 (Figure 2A-F). Results from the Infinium Methylation EPIC BeadChip DNA methylation analysis for the ATRT and the ependymoma did not assign a molecular diagnosis in the online Heidelberg classifier tool ${ }^{2}$. However, $t$ -SNE plotting associated the ATRT with the clinically favorable subclass of ATRT-TYR (Figure 2G) and the ependymoma with the myxopapillary subclass (Figure 2I). Copy number profiles confirmed a heterozygous loss of Chr 22 in both tumors (Figure 2H, J). In line MLPA detected large deletions of SMARCB1 in both tumors and indicated, that the allele containing the exon 6 gain was retained in the tumors whereas the wild-type allele was deleted. No additional SMARCB1 mutation was found in the HCL-v of II.2 .

\section{Discussion}

RTPS1 is characterized by early-onset and even congenital ATRT, and less commonly extracranial rhabdoid tumors. Synchronous or metachronous tumor sequences occur. Thus, RTPS1 is a negative prognosticator in ATRT. Mutations in RTPS1 are usually truncating mutations in distinction to those in Schwannomatosis or Coffin-Siris syndrome ${ }^{1}$. However, overlap between Schwannomatosis and ATRT has been observed ${ }^{3}$. We here report a family in which a gain of SMARCB1 exon 6 segregates with malignant diseases including ATRT. Though we formally cannot rule out an integration of the gained segment elsewhere in the genome, the combination of germline and somatic analyses strongly suggest this change to be pathogenic to one allele of $S M A R C B 1$, which is retained in the tumors lacking INI1 staining. Interestingly, SMARCB1 exon 6 duplications have previously been reported in a pedigree with ATRT and schwannoma ${ }^{4}$. Another pedigree with a CRINET and incomplete penetrance of germline $S M A R C B 1$ exon 6 duplication is on record ${ }^{5}$.

Our index patient III.1, his sister III.2, and his father II.2 did not present any schwannoma on clinical or MRI examination. One cousin IV.1 died from a malignant brain tumor in infancy. Her mother III.4 is an asymptomatic carrier of theSMARCB1 gain, it is possible that she was also afflicted with an ATRT. Regarding the many siblings and half siblings of the father we could not accrue more information. Thus, the exact incidence of further neoplasm was not ascertained and the founding mutation could not be traced back.

A differential diagnosis of a CRINET was entertained for III.1, as CRINETs may be similar to ATRT-TYR on methylation profiling, immunochemistry, and by clinical outcome. However, the absence of any cribriform features and rosettes combined with an abundance of typical rhabdoid cells did not support CRINET as a differential diagnosis. No rhabdoid features were seen in the myxopapillary ependymoma ofII.2 . DNA methylation profiling did neither assign a significant score for the ATRT nor for the myxopapillary ependy- 
moma on the Heidelberg classifier. While this is a frequent phenomenon for tumors with germline alterations (unpublished observations), thet -SNE suggested the ATRT to belong to the prognostically favorable TYR subgroup, in line with the long-term remission observed in our patient. The spinal tumor showed, both histologically and regarding DNA methylation high similarity to the subgroup of myxopapillary ependymoma, a subgroup that is usually occurring in a sporadic setting.

Apart from the myxopapillary ependymoma, the father suffered from a HCL-v. Classical hairy cell leukemia (HCL-c) is typically associated with oncogenic BRAF V600E mutations. Epigenetic driver mutations affecting members of the SWI/SNF complex (e.g. ARID1A ) have been detected in some of those HCL-c, but at increased frequency in hairy cell leukemia variants (HCL-v) that occur without the BRAF V600E mutation. ${ }^{6,7}$ However, both ependymoma and HCL-v have so far not been observed in the context of SMARCB1 mutations.

Together, our report highlights the incomplete penetrance of RTPS1 caused by SMARCB1 gains and the expanding spectrum of malignancies associated with these mutations.

\section{Acknowledgements}

U.S. was supported by the Deutsche Forschungsgemeinschaft and the Fördergemeinschaft Kinderkrebszentrum Hamburg. This work was supported by grants 70112697 and 70114478 from Deutsche Krebshilfe.

Part of this work was a poster presentation at the 2019 Annual Meeting of the German Society for Neuropathology and Neuroanatomy (DGNN) in Magdeburg

\section{References}

1. Holsten T, Bens S, Oyen F, et al. Germline variants in SMARCB1 and other members of the BAF chromatin-remodeling complex across human disease entities: a meta-analysis. Eur J Hum Genet.2018;26(8):1083-1093.

2. Capper D, Jones DTW, Sill M, et al. DNA methylation-based classification of central nervous system tumours. Nature.2018;555(7697):469-474.

3. Kehrer-Sawatzki H, Kordes U, Seiffert S, et al. Co-occurrence of schwannomatosis and rhabdoid tumor predisposition syndrome 1. Mol Genet Genomic Med. 2018.

4. Swensen JJ, Keyser J, Coffin CM, Biegel JA, Viskochil DH, Williams MS. Familial occurrence of schwannomas and malignant rhabdoid tumour associated with a duplication in SMARCB1. J Med Genet.2009;46(1):6872 .

5. Ibrahim GM, Huang A, Halliday W, et al. Cribriform neuroepithelial tumour: novel clinicopathological, ultrastructural and cytogenetic findings. Acta Neuropathol. 2011;122(4):511-514.

6. Maitre E, Bertrand P, Maingonnat C, et al. New generation sequencing of targeted genes in the classical and the variant form of hairy cell leukemia highlights mutations in epigenetic regulation genes. Oncotarget. 2018;9(48):28866-28876.

7. Swerdlow SH, Campo E, Pileri SA, et al. The 2016 revision of the World Health Organization classification of lymphoid neoplasms. Blood. 2016;127(20):2375-2390.

\section{Legends}

\section{Figure 1: Pedigree of described kindred and summary of molecular results}

Individuals with malignant CNS tumors and leukemia are highlighted in $\mathbf{A}$. The index patient is marked by an oblique arrow. Confirmed SMARCB1 exon 6 gain carriers (gain+) and non-carriers (gain-) are identified. Carriers III.2 and III.4 are unaffected at age 38 and 33 years. Specimens from individual IV.1 were not available. Molecular results from available samples are summarized inB . MLPA, multiplex ligation-dependent probe amplification; $t$-SNE, $t$-distributed stochastic neighbour embedding 


\section{Figure 2: Analysis of CNS tumors from II.2 and III.1}

Both ATRT (A,C ) and ependymoma (B,E ) display typical histology with rhabdoid features $(\mathbf{C})$ or Astra-positive matrix, respectively, (E ) as hallmark features. Both tumors were negative for SMARCB1 by immunohistochemistry $(\mathbf{D}, \mathbf{F})$ with arrows indicating non-neoplastic ependymal cells as an internal positive control. Analysis of the DNA methylation profiles revealed a classifier score of 0.48 for the ATRT (0.38 for the TYR-subclass, not shown) and a score $<0.3$ for the ependymoma (not shown). Using $t$-SNE together with $>2500$ cases from 82 brain tumor classes from Capper et al. ${ }^{1}$, the ATRT clustered with the Tyrosinase subclass (zoom-in shown in $\mathbf{G}$ ) and the ependymoma with the myxopapillary subclass (zoom-in shown in $\mathbf{I}$ ). Copy number plots are shown in $(\mathbf{H})$ and $(\mathbf{J})$. Scale bar in Acorresponds to $100 \mu \mathrm{m}$ in A, B . Scale bar in $\mathrm{C}$ corresponds to $10 \mu \mathrm{m}$ in $\mathbf{C - F}$.

\section{Hosted file}

Figure 1.pdf available at https://authorea.com/users/395744/articles/508887-evidence-for-alow-penetrant-extended-phenotype-of-rtps1-from-a-kindred-with-gain-of-smarcb1-exon- 6

\section{Hosted file}

Figure 2.pdf available at https://authorea.com/users/395744/articles/508887-evidence-for-alow-penetrant-extended-phenotype-of-rtps1-from-a-kindred-with-gain-of-smarcb1-exon- 6 